\title{
Adaptive Radiation Therapy for Prostate Cancer
}

\author{
Michel Ghilezan, M.D, Ph.D, Di Yan, D.Sc, and Alvaro Martinez, M.D \\ Department of Radiation Oncology, William Beaumont Hospitals and Research Institute, Royal \\ Oak, MI 48073.
}

\section{Abstract}

Adaptive radiotherapy has been introduced to manage an individual's treatment by, including patient-specific treatment variation identified and quantified during the course of radiotherapy in the treatment planning and delivering optimization. Early studies have demonstrated that this technique could significantly improve the therapeutic ratio by safely reducing the large target margin that has to be used in conventional radiotherapy for prostate cancer treatment. Clinical application of off-line image-guided adaptive radiotherapy for prostate cancer has demonstrated encouraging clinical outcome. Long-term clinical follow-up has shown significant improvement in terms of tumor control and low toxicity profile, emphasizing the beneficial effect of imageguidance and adaptive treatment. Continuous development in adaptive radiotherapy has made possible additional increases in target dose by further reducing target margin when using online image-guided adaptive intensity-modulated radiation therapy. However, clinical implementation of new techniques should be explored cautiously and should include a comprehensive management strategy to address uncertainties in target definition and delineation in the preclinical implementation studies.

\section{Introduction}

Conformal radiation therapy (CRT) and intensity-modulated radiation therapy (IMRT) are used routinely in prostate cancer radiation treatment in an effort to maximize the therapeutic ratio. The success of these techniques relies on the accurate delivery of radiation dose. However, treatment optimization is limited by variation in delivered dose caused by errors in patient treatment positioning, inter-treatment, and intra-treatment variation of organ position, size, and shape. Clinical studies [1,2] have shown the negative consequences in terms of target coverage and clinical outcome of using CRT or IMRT without properly accounting for patient and organ variations during the course of radiotherapy. The conventional method to compensate for variations in treatment position and internal organ motion typically involves adding a large margin around the target, thereby increasing the treatment volume and consequently increasing normal organ toxicity while limiting the tumor dose.

Adaptive radiotherapy has been introduced to either reduce or compensate for the effect of patient-specific treatment variation measured during the course of radiotherapy [3]. To understand and promote clinical implementation of adaptive radiotherapy for prostate cancer, this article will review the existing adaptive techniques, as well as the corresponding clinical trials and treatment outcome. In addition, recent advances in adaptive radiotherapy techniques enabled by new technologies in imaging, planning, and delivery will be also overviewed and discussed.

Corresponding Author: Michel Ghilezan, MD, PhD, Department of Radiation Oncology, William Beaumont Hospitals and Research Institute, Royal Oak, MI 48073-6769. mghilezan@ beaumont.edu. 


\section{Conventional Management of Treatment Position Variation}

A major source of treatment position uncertainty is the variation in the daily setup of the patient. A common practice to minimize setup error is weekly evaluation of port film. This has been shown to be insufficient. 4 The use of immobilization devices is also common and should, in theory, enhance the reproducibility of daily patient setup. However, studies of their effectiveness for patient treated on thoracic and abdominal regions have produced variable results [5-8].

Two approaches to either compensate for or reduce the magnitude of patient setup errors have been developed in previous studies. The first approach incorporates generic setup errors, characterized from measurements made on the patient population and incorporated into the initial treatment planning through a target margin [9-12]. The second approach uses a decision rule for setup adjustments to reduce the magnitude of setup error and to minimize the frequency of patient repositioning [13-16]. The decision rule approach requires more frequent portal imaging and complements the use of electronic portal imaging devices. However, the setup adjustment is not incorporated into treatment planning, and potential changes in the dose delivered to the individual patient are not considered. More importantly, both approaches employ average values derived from population studies in the target margin design; therefore, the opportunity to optimize the individual's treatment is not exploited.

The other major source of treatment position uncertainty in prostate cancer radiotherapy is internal organ variation in position, shape, and size. Inter-and intra-treatment variations of prostate and seminal vesicles in position and shapes have been traditionally compensated for by adding generic margins to the clinical target volume (CTV) at the time of pretreatment planning. The proper margin to be added to the CTV has been a matter of debate. Although some reports recommended that about $10-\mathrm{mm}$ margin in the anterior- posterior direction to cover 95\% of the uncertainty in setup and organ motion [17], other studies suggested that margins over $10 \mathrm{~mm}$ should be added to CTV, especially if extra-capsular invasion was suspected [18]. Dearnaley et al reported the results of a randomized trial of 2 radiation dose levels (conventional and high-dose) and 2 margins, 10 or $15 \mathrm{~mm}$, around the CTV. Their data demonstrated equivalent tumor control achieved using either 10-mm margin or 15-mm margin, suggesting that a 10-mm margin may be sufficient for CRT.19 However, as has been pointed out, 20 the consequence of target under-dose as a result of treatment position variation in a relatively small group of patients $(10 \%-20 \%)$ cannot likely be evaluated using the actual clinical outcome data unless under-dosing is severe for most patients. Therefore, the appropriateness of a generic planning target margin, although a very important clinical quality assurance issue, may not be testable with existing clinical data or using a clinical trial.

De Crevoisier et al [1] demonstrated the risks of under-dosing the target with dramatic consequences in terms of biochemical control (BC) and local failure of prostate cancer patients treated with definitive CRT. The patients were positioned according to skin tattoos and bony landmarks on weekly portal films. The hypothesis of their study was that if the rectum was distended on planning computed tomography $(\mathrm{CT})$, there was a high probability that it would be less distended during the treatment course and therefore the target (prostate) would shift to posterior on average, relative to the planning CT position. The result would be target under-dosing and a relative larger sparing of rectal wall, leading to decreased biochemical and/or local control as well as improved rectal toxicity. Indeed, patients with distended rectums on planning CT showed almost 30\% decrease in their BC at 5 years and the reduction was even higher for intermediate-and high-risk patients. Likewise, there was a significant lower incidence of grade 2 rectal bleeding in the group of patients with a distended rectum on planning CT because of the inclusion of less rectal volume on average 
in the high-dose region during the treatment course. The negative effect on biochemical and local control of the planning CT rectal distension status was confirmed in a Dutch study reported by Heemsbergen et al. [2]. They found a reduction of $20 \%$ in the freedom from failure rate in a subgroup of patients with larger ano-rectal volumes. These 2 studies underline not only the negative impact on target dosimetric coverage, if treatment planning is performed on a single CT image set that may not be representative for the patient internal anatomy throughout the treatment course, but also the significant detrimental effect on clinical outcome.

In summary, the use of $\geq 10 \mathrm{~mm}$ generic margin would deliver large doses to the organs at risk, resulting in increased toxicity and limiting the potential for dose-escalation with CRT or IMRT. Nevertheless, the large generic target margin cannot be safely reduced using the conventional managements for prostate cancer radiotherapy.

\section{Clinical Implementation of Off-Line Image-Guided Adaptive Radiotherapy}

In the mid-1990s, an off-line adaptive process was developed to minimize the deleterious effects of treatment position uncertainty on each individual patient treatment.21-23 A process (Fig. 1) was implemented in which the treatment plan of the individual patient was adaptively modified part way through the treatment, on the basis of time course of setup variation measured by daily portal images and internal organ motion captured by serial CT measurements.22 The treatment was initiated with a conventional 4-field CRT technique for the first 5 fractions. Online portal imaging and off-line CT scans were obtained for each of the first 4 treatment days. Portal images were analyzed to evaluate patient-specific systematic and random setup errors based on bony anatomical landmarks. The target (prostate \pm seminal vesicles) was contoured on the serial CT images that were previously registered based on bony anatomy to the planning $\mathrm{CT}$ image, to determine the target motion or deformation. A new patient-specific planning target volume (PTV) was constructed, which eliminated the systematic variation and compensated for individual random variation estimated using all the measurements obtained during the previous treatments. Statistic confidence and achievable tolerance of using patient-specific PTV in treatment has been evaluated (Fig. 2) and reported in an early publication [22]. The expected number of treatment CT scans used in the PTV construction was predetermined before the clinical implementation. By taking advantage of the computer-controlled multi-leaf collimator (MLC), a second, modified treatment plan was calculated and implemented by adjusting the beam aperture or intensity through the inverse planning, and selecting a proper prescription dose level simultaneously for the remaining CRT or IMRT treatments. The prescription dose of the individual patient was selected, with respect to the low-, intermediate-, or high-risk of prostate cancer, on the basis of predefined rectal wall and bladder dose or volume constraints. A follow-up study 23 on this adaptive process demonstrated that the prescription dose level for the majority of the patients could be increased up to $10 \%$ (mean $=5 \%$ ) or 1.8-7.2 Gy (mean $=3.6 \mathrm{~Gy}$ ), when compared with the conventional CRT treatment process. That level could be further increased to $5 \%-15 \%$ (mean $=7.5 \%$ ) or $3.2-10.8$ Gy (mean $=5.4$ Gy), when the IMRT delivery was used in the adaptive process. Figure 3 shows the distributions of prescription dose for the patients treated using either a 4-field CRT or a 5beam IMRT in the off-line adaptive process. The study also indicated that at least $10 \%$ of patients could have had potential target miss when treated in the conventional treatment process. An update on 263 patients treated with the adaptive process showed that only 12 of 263 patients or $4.6 \%$ had a portion of their seminal vesicles missed and 5 of 263 patients or $1.9 \%$ had a portion of their prostate missed [24]. Wloch et al. [24] recently reported a quantitative evaluation of quality control in 1017 patients treated with this adaptive process. Only 96 patients (9.4\%) required a second modification of the MLC configuration, including 63 corrections of the residual systematic error in one single direction. In addition, only 5 of 
1017 patients required re-adjustment of the target margins due to a larger random setup error.

A different approach of adaptive radiotherapy has been undertaken by the Netherlands Cancer Institute (NKI). Hoogeman et al. [25] used CT data to evaluate an adaptive offline procedure that calculated both an average prostate position and rectum shape based on the planning CT and the first 4 repeat CT scans. Using this methodology, the systematic variation in the position and shape of the rectal wall was also considered in the adaptive planning modification. In a follow-up study, the NKI team was able to demonstrate a PTV margin reduction from 10 to $7 \mathrm{~mm}$ without compromising target coverage and in the same time, reducing the rectal wall dose by about 30\%.26 Recently, Nijkamp et al. [27] published the first clinical results of the NKI adaptive protocol. They used weekly cone-beam CT images rather than conventional CT scans as basis for their off-line adaptive strategy. Specific dietary instructions and mild laxatives were recommended to keep the rectum empty thus minimizing rectal and target inter-fraction and intra-fraction motion. A total of 20 prostate cancer patients were included in the study. An average PTV reduction of $29 \%$ was achieved. The volume of the average rectal wall that received $>65 \mathrm{~Gy}$ was reduced by $19 \%$.

The recent development in off-line adaptive radiotherapy is the adaptive inverse planning [28-31]. By including the delivered dose and the variation of organs of interest directly in the planning objective function, the dose distribution accomplished in the adaptive inverse planning is optimized for the remaining treatments. A retrospective study [32] on prostate cancer patients with multiple daily CT scans has shown that $10 \%$ gain in therapeutic ratio could be achieved with respect to conventional inverse planning when the adaptive inverse planning with a single planning modification was applied after the first week of the treatment. In addition, Birkner et al. [28] demonstrated that the single modification was comparable to the multiple modifications with respect to the final treatment dose distribution. To date, adaptive inverse planning for off-line plan modification has not been implemented routinely in the clinic because of the unreliability of CT image based deformable organ registration. Baum et al. [31] suggested an alternative approach that performs adaptive inverse planning using coverage probability, instead of using the organ sub-volume variation distribution determined using deformable organ registration. On the basis of their study, using coverage probability in the adaptive inverse planning could achieve comparable results to those achieved by using the organ sub-volume variation distribution.

\section{Clinical Outcome of Off-Line Image-Guided Adaptive Radiotherapy}

Clinical results on the off-line adaptive treatment have been reported by the Beaumont group [33-39]. The effect and tremendous gain in BC from dose escalation enabled by high precision and accurate radiotherapy shown in Fig. 4 reflects the continuum improvement on $\mathrm{BC}$ of 3064 prostate cancer patients in a single clinic treated during the prostate-specific antigen (PSA) era [38]. Patients with stage T1-3 N0 M0 prostate cancer were treated with either low dose external beam radiotherapy with median of $66.6 \mathrm{~Gy}(\mathrm{n}=1199$ patients), high dose adaptive CRT or IMRT with a median of $79.6 \mathrm{~Gy}(\mathrm{n}=1024$ patients), or high dose brachytherapy with or without external beam radiotherapy $(n=841$ patients). BC was scored according to the Phoenix definition. The 5-year BC was $51 \%$ for patients treated in $1987-1990,62 \%$ for $1991-1995,83 \%$ for $1996-2000$, and $90 \%$ for $2001-2005(p<.01)$. The 5 -year clinical failure rate as reported was $24 \%$ for patients treated in $1987-1990,14 \%$ for 1991-1995, 4\% for 1996-2000, and 2\% for 2001-2005 ( $p<.01)$. Similar findings were noted for improved overall and cause-specific survival. Although the follow-up for patients treated 
by the offline adaptive CRT or IMRT during 2001-2005 was shorter, the trend of BC improvement was significant.

In one of their latest report [39], they evaluated the acute and chronic toxicity of 642 patients treated in the adaptive process with a minimum follow-up of 4 years. For each the patients, a prescription dose level were selected within the range of 70.2-79.2 Gy to the patient-specific PTV or 74-84 Gy to the isocenter based on rectal wall and bladder dose-volume histograms (DVHs) constraints. The treatment was delivered with 4-field CRT before the end of 2003 and 5-beams IMRT thereafter. The toxicities observed were similar within the various dose levels used and equal or lower to those reported by other institutions that have been dose escalating.40-42 Nonetheless, the prostate gland doses selected by the adaptive process were on the high end of the spectrum with isocenter International Commission on Radiation Units and Measurements doses in the range of 74-84 Gy. Tables 1 and 2 show the grade 2 and grade 3 chronic toxicities of these patients stratified by the dose levels in low (70.2-72 Gy), intermediate (72-75.6 Gy), and high dose (75.6-79.2). Most common grade 2 genitourinary (GU) and gastrointestinal (GI) chronic toxicities were urinary frequency or urgency, rectal bleeding, and proctitis; however, the occurrence of these side-effects were low compared with other dose escalation series. Grade 3 toxicities, mostly urinary retention and rectal bleeding were also infrequent, generally below 5\%. Most importantly, in this large cohort of patients, there were no statistically significant differences between the chronic toxicity profiles across the 3 dose levels, confirming the preliminary results and demonstrating that high prescription doses can be safely delivered to the prostate using the off-line adaptive process. In addition, the low rectal and bladder chronic toxicity rates reflected primarily the DVH constraints cutoffs used rather than the actual treatment dose levels. Therefore, these results demonstrate that the margin reduction achieved with the adaptive process, with the corresponding improvement in accuracy and precision of dose delivery, has resulted in significant dose escalation while maintaining low genitourinary and gastrointestinal isotoxicity levels.

The off-line adaptive treatment with IMRT shows extra clinical benefit. Martinez et al37 compared the adaptive CRT technique (556 patients) with the adaptive IMRT technique (172 patients) applied in the adaptive process. Table 3 shows the results with the median follow-up 4.3 vs 2.2 years for the adaptive CRT and the adaptive IMRT groups, respectively $(p<.01)$. The adaptive CRT patients experienced significantly higher acute grade $\geq 2$ urinary retention ( $7 \%$ vs $2 \%$ for the adaptive IMRT, $p=.03$ ) as well as higher acute grade $\geq 2$ rectal pain or tenesmus ( $19 \%$ vs $5 \%$ for the adaptive IMRT, $p<.01)$. Chronic genitourinary and GI toxicity were generally low in both groups. However, 17 patients (3\%) in the adaptive CRT group developed grade $\geq 2$ chronic urinary retention vs only 1 patient $(0.5 \%)$ in the adaptive IMRT group $(p=.05)$. More importantly, 86 patients $(16 \%)$ treated with the adaptive CRT developed grade $\geq 2$ chronic rectal bleeding vs 6 patients (4\%) in the adaptive IMRT group $(p<.01)$. The median time to rectal bleeding was 1.0 year for the adaptive CRT vs 0.9 year for the adaptive IMRT. The actuarial 1,2 , and 3 year grade $\geq 2$ chronic rectal bleeding was $6 \%, 17 \%$, and $18 \%$ for the adaptive CRT vs $3 \%, 3 \%$, and $5 \%$ for the adaptive IMRT, respectively $(p<.01)$. Of significance was that there were more group II patients (intermediate/high-risk patients in whom the proximal $2 \mathrm{~cm}$ of seminal vesicles are treated, resulting in larger target volumes) treated with the adaptive IMRT $61 \%$ vs $51 \%$ for the adaptive CRT $(p<.01)$ and yet the toxicity levels observed were lower with the adaptive IMRT.

The BC using the Phoenix definition was reported (Table 4) in a review of 844 patients treated with the adaptive process.39 There were significant survival outcome differences between group I (favorable-risk) and group II (intermediate- or high-risk) patients. This is an expected finding since these patients have a worse prognosis. The 5-year outcomes for 
group II are very encouraging. In addition, stratifying these patients into 2 groups by their rectal volume manifested on the planning CT image, the BC, chronic GI toxicity and the rates of chronic grade $\geq 2$ rectal bleeding were similar between the 2 groups thus underlining the significant reduction in target miss [43]. In contrast, this target miss has occurred, as reported before [1,2], because of the distended rectum on planning CT and brought great concern to the conventional management of prostate cancer radiotherapy.

In summary, off-line image-guided adaptive prostate radiotherapy has been implemented in a few clinics. So far, about 2000 patients have been successfully treated using this technique. With adequate follow-up, significant improvements in both normal tissue toxicity and tumor control have been reported, validating the adaptive process and therefore justifying the routine implementation of this technique in radiation oncology facilities.

\section{Recent Advances in Online Image-Guided Adaptive Radiotherapy}

Clinical implementation and development of adaptive radiotherapy for prostate cancer have been greatly promoted by onboard volumetric imaging technology, specifically the onboard cone beam CT (CBCT) technology [44,45]. Using onboard imaging, treatment target and the adjacent anatomy can be localized for a patient in the treatment position before treatment delivery and even simultaneously during the treatment delivery. This enables online correction and adaptive treatment in routine clinical operations. Letourneau et al. [46] evaluated the accuracy and precision of an online CBCT image guidance system, and demonstrated the system accuracy from CT simulation planning to beam delivery to be within $1 \mathrm{~mm}$. However, the soft-tissue target registration error was large with the mean and the standard deviation $-1.1 \pm 2.9 \mathrm{~mm}$ in patient superior-inferior direction.

Online volumetric image-guided adaptive techniques have been fairly broad, including online re-planning through direct beam aperture modification for CRT [47] or MLC segment adjustment for IMRT [48], online inverse planning [49-51], hybrid online correction or offline replanning [52,53], and full online adaptive inverse planning [54,55]. Online beam aperture modification adapts the field shape to the online measurement of prostate and seminal vesicle deformation, whereas MLC segment adjustment adapts the MLC segments to the geometrical changes of both target and critical organs manifested on the daily volumetric image. A recent study [56] shows that seminal vesicles move significantly more in comparison with the prostate. Consequently, 50\% extra target margin is required in the online image-guided target localization and correction for the seminal vesicles, compared with the prostate. Online beam aperture or MLC segment modification improves target dose coverage compared with the online rigid body correction, which in principle is also a more reliable correction method in clinical practice, but is still limited in its clinical implementation because of the additional burden of treatment plan approval process.

Ghilezan et al [50] performed a benchmark study to assess the potential benefit of online inverse planning, and concluded that a target dose increase of $13 \%$ ( $\mathrm{SD}=9.7 \%$ ), on average, could be achieved with online inverse planning based on the equal biologically equivalent dose of the rectal wall, and $53.3 \%$ ( $\mathrm{SD}=15.3 \%$ ) based on the equal biologically equivalent dose of the bladder wall. However, the variation ( $\mathrm{SD}=9.7 \%$ or $15.3 \%$ ) was fairly large among patients, $27 \%$ of patients had only minimal benefit ( $<5 \%$ of dose increment) from the online inverse planning and $32 \%$ had significant benefit (>15\%-41\% of dose increment). The online inverse planning results were confirmed by another recent study [51]. This study demonstrated that an extra $4 \%$ improvement in dose escalation could be achieved by the online inverse planning compared with the online target position correction alone. However, both techniques adapt the instant variation manifested on a CBCT image only, the effect of intra-treatment variation and the effect of dose variation delivered in the previous treatments 
are not considered. Justus et al [53] analyzed intra-treatment target motion of 22 patients treated in a clinical protocol of online CBCT image-guided radiotherapy and found that $77 \%$ (17 of 22) of patients had relatively small intra-fraction motion requiring $3 \mathrm{~mm}$ target margins in both anterior-posterior and superior-inferior directions, but $23 \%$ (5 of 22 ) of patients had relatively large motion requiring the target margins of $6 \mathrm{~mm}$ on both the directions. In addition, target margins were similar about $2 \mathrm{~mm}$ in the right-left direction for all patients. On the basis of the study, they proposed an online/off-line "hybrid" image guidance technique, where online target localization and position correction were performed daily, whereas the individual target margin would be modified using the intra-treatment measurements obtained during the first 4 fractions, and the pretreatment plan would be modified on the new patient-specific PTV for the rest of treatments. In fact, the most sophisticated online adaptive planning is the online adaptive inverse planning. Using both pre-and post-treatment volumetric images, online adaptive inverse planning technique [54] can be performed to include both the intra-treatment variation and the delivered dose variation, specifically in the normal organs, in the online inverse planning optimization. Using the advantage of relative position between rectal wall and target manifested on the daily image, daily dose per fraction can also be optimized in the adaptive planning optimization [57]. This technique was evaluated [55] in a small group of patients using multiple daily CT images. An additional $3 \%$ therapeutic improvement could be achieved compared with the online inverse planning technique.

New innovations in imaging and delivery technology will continue to improve and promote the online image guidance techniques. Current onboard CBCT imaging is suboptimal for online guidance of prostate cancer treatment due to poor soft tissue contrast. The onboard MRI machine [58] will potentially enhance the online image guidance capability and subsequently improve treatment accuracy. However, the continuous development of online techniques should not be limited to academic interest only; an ambitious and comprehensive approach should also be clinically justifiable. An immediate concern with respect to the reduction of target margin in online image guidance is the uncertainty in the target definition. Chao et al [59] reported a clinic-pathologic analysis of 371 radical prostatectomy specimens on extra-capsular extension (ECE). They found that more than $4 \mathrm{~mm}$ of ECE beyond the prostate capsule might exceed $20 \%$ in patients with a PSA $\geq 10 \mathrm{ng} / \mathrm{mL}$ and biopsy Gleason score $\geq 7$. Applying no gross target volume (GTV)-to-CTV margin, as well as a very small PTV margin (1-3 mm), in the online image-guided treatment is thus questionable. A target with large ECE could be potentially missed during highly accurate delivery using online image guidance. Currently, noninvasive procedures and technologies to measure patient-specific ECE are lacking; the best technique to compensate for this uncertainty is to include a patient population-based ECE distribution in the target margin and dose escalation design. Because the best knowledge of ECE distribution is probabilistic in nature, the potential dose escalation through target margin reduction and the confidence of ECE margin becomes contradictory. Thus, an optimal compromise needs to be investigated before an ambitious approach of margin reduction is implemented.

\section{Conclusions}

A large target margin has been the traditional approach of accounting for treatment position variations in conventional radiotherapy. This has been one of the major limiting factors in the prostate cancer treatment using external beam radiation therapy. The planning target margin can be significantly reduced by systematically accounting for patient-specific variations. However, the management of patient-specific anatomical variation requires multiple measurements of patient anatomy accomplished by different image feedbacks. Among them, volumetric image feedback, (including both off-board conventional CT and onboard CBCT) has been most commonly used. The most effective method in image 
feedback management of radiotherapy is the adaptive control methodology, with the aim to customize each patient's treatment plan to patient-specific variation by evaluating, characterizing, and incorporating the systematic and random variations in the adaptive planning optimization. Long-term clinical follow-up has demonstrated encouraging results in terms of clinical outcome and toxicity profile, underlining the strong clinical effect of image guidance and adaptive treatment. Continuous development in adaptive radiotherapy has shown that an average increase in target dose of, $10 \%$ or higher through additional target margin reduction can be achieved with using online adaptive IMRT. However, clinical implementation of these new techniques should be explored cautiously and should include both uncertainties in target definition and delineation in the pre-implementation studies.

\section{Acknowledgments}

Clinical translational research of adaptive radiotherapy for prostate cancer in Radiation Oncology, William Beaumont Hospitals has been supported in part by NCI, Grants: R29 CA71785, R01 CA118037, R01 CA091020, R21 CA130330, and US Army Medical Research, Grants: PC970201, PC061209.

Department of Radiation Oncology in William Beaumont Hospital receives research and development grants from ELEKTA Oncological System and Philips Radiation Oncological System.

\section{References}

1. De Crevoisier R, Tucker SL, Dong L, et al. Increased risk of biochemical and local failure in patients with distended rectum on the planning CT for prostate cancer radiotherapy. Int J Radiat Oncol Biol Phys. 2005; 62:965-973. [PubMed: 15989996]

2. Heemsbergen WD, Hoogeman MS, Witte MG, et al. Increased risk of biochemical and clinical failure for prostate patients with a large rectum at radiotherapy planning: Results from the Dutch trial of 68 Gy versus 78 Gy. Int J Radiat Oncol Biol Phys. 2007; 67:1418-1424. [PubMed: 17241751]

3. Yan D, Vicini F, Wong J, et al. Adaptive radiation therapy. Phys Med Biol. 1997; 42:123-132. [PubMed: 9015813]

4. Valicenti RK, Michalski JM, Bosch WR, et al. Is weekly port filming adequate for verifying patient position in modern radiation therapy. Int J Radiat Oncol Biol Phys. 1994; 30:431-438. [PubMed: 7928470]

5. Hunt MA, Schultheiss TE, Desobry GE. An evaluation of setup uncertainties for patients treated to pelvic sites. Int J Radiat Oncol Biol Phys. 1995; 32:227-233. [PubMed: 7721620]

6. Soffen EM, Hanks GE, Hwang CC, et al. Conformal static field therapy for low volume low-grade prostate cancer with rigid immobilization. Int J Radiat Oncol Biol Phys. 1990; 20:141-146. [PubMed: 1993623]

7. Song PY, Washington M, Vaida F, et al. A comparison of four patient immobilization devices in the treatment of prostate cancer patients with three-dimensional conformal radiotherapy. Int J Radiat Oncol Biol Phys. 1996; 34:213-219. [PubMed: 12118554]

8. Verhey L. Immobilization and positioning patients for radiotherapy. Semin Radiat Oncol. 1995; 5:100-114. [PubMed: 10717133]

9. Goitein M. Calculation of the uncertainty in the dose delivered during radiation therapy. Med Phys. 1985; 12:608-612. [PubMed: 4046996]

10. Kutcher GJ, Mageras GS, Leibel SA. Control, correction, and modeling of setup errors and organ motion. Semin Radiat Oncol. 5:134-145. [PubMed: 10717135]

11. Leong J. Implementation of random positioning error in computerized radiation treatment planning systems as a result of fractionation. Phys Med Biol. 1987; 32:327-334. [PubMed: 3575415]

12. Urie MM, Goitein M, Doppke K, et al. The role of uncertainty analysis in treatment planning. Int J Radiat Oncol Biol Phys. 1991; 21:91-107. [PubMed: 1903372]

13. Bel A, Van Herk M, Bartelink H, et al. A verification procedure to improve patient setup accuracy using portal images. Radiol Oncol. 1993; 29:253-260. 
14. Denham JW, Dally MJ, Hunter K, et al. Objective decision-making following a portal film: The results of a pilot study. Int J Radiat Oncol Biol Phys. 1993; 26:869-876. [PubMed: 8344856]

15. Dutreix A, van der Schueren E, Leunens L. Quality control at the patient level: Action or retrospective introspection. Radiol Oncol. 1992; 25:146-147.

16. Yan D, Wong J, Gustafson G, et al. A new model for "accept or reject" strategies in off-line and on-line megavoltage treatment evaluation. Int J Radiat Oncol Biol Phys. 1995; 31:943-952. [PubMed: 7860410]

17. Tinger A, Michalski JM, Cheng A, et al. A critical evaluation of the planning target volume for 3D conformal radiotherapy of prostate cancer. Int J Radiat Oncol Biol Phys. 1998; 42:213-221. [PubMed: 9747840]

18. Rasch C, Steenbakkers R, Van Herk M. Target definition in prostate, head, and neck. Semin Radiat Oncol. 2005; 15:135-145.

19. Dearnaley DP, Hall E, Lawrence D, et al. Phase III pilot study of dose escalation using conformal radiotherapy in prostate cancer: PSA control and side effects. Br J Cancer. 2005; 92:488-498. [PubMed: 15685244]

20. Niemierko A. Target dose inhomogeneity and the location recurrence. Int J Radiat Oncol Biol Phys. 2002; 54:128.

21. Yan D, Wong J, Vicini F, et al. Adaptive modification of treatment planning to minimize the deleterious effects of treatment setup errors. Int J Radiat Oncol Biol Phys. 1997; 38:197-206. [PubMed: 9212024]

22. Yan D, Lockman D, Brabbins D, et al. An off-line strategy for constructing a patient-specific planning target volume in adaptive treatment process of prostate cancer. Int J Radiat Oncol Biol Phys. 2000; 48:289-302. [PubMed: 10925000]

23. Martinez A, Yan D, Brabbins D, et al. Improvement in dose escalation using the process of adaptive radiotherapy combined with 3D-conformal or intensity modulated beams for prostate cancer. Int J Radiat Oncol Biol Phys. 2001; 50:1226-1234. [PubMed: 11483333]

24. Wloch J, Yan D, Brabbins D, et al. Quality control for image guided radiotherapy prostate cancer (abstr). Int J Radiat Oncol Biol Phys. 2007; 69:S20. (suppl).

25. Hoogeman MS, van Herk M, de Bois J, et al. Strategies to reduce the systematic error due to tumor and rectum motion in radiotherapy of prostate cancer. Radiother Oncol. 2005; 74:177-185. [PubMed: 15734206]

26. Nuver TT, Hoogeman MS, Remeijer P, et al. An adaptive offline procedure for radiotherapy of prostate cancer. Int J Radiat Oncol Biol Phys. 2007; 67:1559-1567. [PubMed: 17306934]

27. Nijkamp JN, Pos FJ, Nuver TT, et al. Adaptive radiotherapy for prostate cancer using kilovoltage cone-beam computed tomography: First clinical results. Int J Radiat Oncol Biol Phys. 2008; 70:75-82. [PubMed: 17869445]

28. Birkner M, Yan D, Markus A, et al. Adapting inverse planning to patient and organ geometrical variation: Algorithm and implementation. Med Phys. 2003; 30:2822-2831. [PubMed: 14596318]

29. Rehbinder H, Forsgren C, Lof J. Adaptive radiation therapy for compensation of errors in patient setup and treatment delivery. Med Phys. 2004; 31:3363-3371. [PubMed: 15651619]

30. Unkelbach J, Oelfke U. Incorporating organ movements in IMRT treatment planning for prostate cancer: Minimizing uncertainties in the inverse planning process. Med Phys. 2005; 32:2471-2483. [PubMed: 16193776]

31. Baum C, Alber M, Birkner M. Robust treatment planning for intensity-modulated radiotherapy of prostate cancer based on coverage probabilities. Radiother Oncol. 2006; 78:27-35. [PubMed: 16216359]

32. Yan D, Birkner M, Nuesslin F. Improvement in dose escalation using off-line and on-line image feedback in the intensity modulated beam design for prostate cancer treatment (abstr). Int J Radiat Oncol Biol Phys. 2001; 51:91. (suppl).

33. Brabbins D, Martinez A, Yan D, et al. A dose escalation trial using the adaptive radiotherapy process (ART) as a delivery system in localized prostate cancer: Analysis of chronic toxicity. Int J Radiat OncolBiol Phys. 2005; 61:400-408. 
34. Vargas C, Kestin L, Yan D, et al. Dose-volume analysis of predictors for chronic rectal toxicity following treatment of prostate cancer with adaptive image-guided radiotherapy. Int J Radiat Oncol Biol Phys. 2005; 62:1297-1308. [PubMed: 16029785]

35. Vargas C, Martinez AA, Yan D, et al. A phase II dose escalation study to image-guided adaptive radiotherapy for prostate cancer: Use of dose constraints to achieve rectal isotoxicity. Int J Radiat Oncol Biol Phys. 2005; 63:141-149. [PubMed: 16111582]

36. Harsolia A, Vargas C, Yan D, et al. Predictors for chronic urinary toxicity after the treatment for prostate cancer with adaptive 3-D conformal radiotherapy: Dose-volume analysis of a phase II dose escalation study. Int J Radiat Oncol Biol Phys. 2007; 69:1100-1109. [PubMed: 17967304]

37. Martinez A, Yan D, Brabbins D, et al. Acute and chronic toxicity of adaptive image guided radiation therapy delivery with 3D-conformal technique vs. intensity modulation in 728 prostate cancer patients (abstr). Int J Radiat Oncol Biol Phys. 2007; 69:S317. (suppl).

38. Brabbins D, Kestin L, Yan D, et al. Improvement in clinical outcomes with prostate radiotherapy at a single institute in the PSA era (abstr). Int J Radiat Oncol Biol Phys. 2008; 72:S318. (suppl).

39. Ghilezan, M.; Yan, D.; Martinez, A. Image-guided radiation therapy in prostate cancer: William Beaumont experience. In: Valicenti, RK.; Dicker, AP.; Jaffray, DA., editors. Image-guided Radiation Therapy of Prostate Cancer. Information Healthcare; New York, NY: 2008. p. 113-126.

40. Zelefsky MJ, Leibel SA, Gaudin PB, et al. Dose escalation with three-dimensional conformal radiation therapy affects the outcome in prostate cancer. Int J Radiat Oncol Biol Phys. 1998; 41:491-500. [PubMed: 9635694]

41. Pollack A, Zagars GK, Smith LG, et al. Preliminary results of a randomized radiotherapy doseescalation study comparing 70 Gy with 78 Gy for prostate cancer. J Clin Oncol. 2000; 18:39043911. [PubMed: 11099319]

42. Horwitz E, Hanlon A, Pinover W, et al. Defining the optimal radiation dose with three-dimensional conformal radiation therapy for patients with non-metastatic prostate carcinoma by using recursive partitioning techniques. Cancer. 2001; 92:1281-1287. [PubMed: 11571744]

43. McGrath S, Kestin L, Dilworth J, et al. Adaptive image-guided radiotherapy to correct for rectal distention in prostate cancer: Five-year biochemical and rectal toxicity outcomes (abstr). Int J Radiat Oncol Biol Phys. 2008; 72:S324. (suppl).

44. Jaffray, DA.; Wong, JW. Exploring "target of the day" strategies for a medical linear accelerator with cone-beam CT scanning capabilities.. Presented at the Proceedings of the XII International Conference on the Use of Computers in Radiation Therapy; Heidelberg, Germany. 1997; p. 172-175.

45. Jaffray DA, Drake DG, Moreau M, et al. A radiographic and tomographic imaging system integrated into a medical linear accelerator for localization of bone and soft-tissue targets. Int $\mathbf{J}$ Radiat Oncol Biol Phys. 1999; 45:773-789. [PubMed: 10524434]

46. Letourneau D, Martinez A, Lockman D, et al. Assessment of residual error for online cone-beam CT-guided treatment of prostate cancer patients. Int J Radiat Oncol Biol Phys. 2005; 62:12391246. [PubMed: 15913917]

47. Feng Y, Castro-Pareja C, Shekhar R, et al. Direct aperture deformation: An inter-fraction image guidance strategy. Med Phys. 2006; 33:4490-4498. [PubMed: 17278800]

48. Court LE, Dong L, Lee AK, et al. An automatic CT-guided adaptive radiation therapy technique by online modification of multileaf collimator leaf positions for prostate cancer. Int J Radiat Oncol Biol Phys. 2005; 62:154-163. [PubMed: 15850916]

49. Wu C, Jeraj R, Olivera GH. Re-optimization in adaptive radiotherapy. Phys Med Biol. 2002; 47:3181-3195. [PubMed: 12361217]

50. Ghilezan M, Yan D, Liang J, et al. Online image-guided intensity-modulated radiotherapy for prostate cancer: How much improvement can we expect? A theoretical assessment of clinical benefits and potential dose escalation by improving precision and accuracy of radiation delivery. Int J Radiat Oncol Biol Phys. 2004; 60:1602-1610. [PubMed: 15590192]

51. Derek S, Liang J, Yan D, et al. Comparison of various online IGRT strategies: The benefits of online treatment plan re-optimization. Radiol Oncol. 2009; 90:367-376.

52. Wu Q, Liang J, Yan D. Application of dose compensation in image-guided radiotherapy of prostate cancer. Phys Med Biol. 2006; 51:1405-1419. [PubMed: 16510952] 
53. Adamson J, Wu Q, Yan D. A hybrid strategy using discriminant analysis for prostate intra-fraction motion management (abstr). Med Phys. 2009; 36:2726.

54. Yan, D.; Birknner, M.; Liang, J., et al. Strategies for off-line and on-line image feedback adaptive radiotherapy. Biological and physical basis of IMRT and Tomotherapy.. Presented at the Proceedings of AAPM Symposium (No. 12); Montreal, Canada. 2002; p. 139-150.

55. Zhang P, Liang J, Yan D. Evaluation of online image guided adaptive inverse planning methodology. Int J Radiat Oncol Biol Phys. 2007; 69:S641. (abstr) (suppl).

56. Liang J, Wu Q, Yan D. The role of seminal vesicle motion target margin assessment for online image-guided radiotherapy for prostate cancer. Int J Radiat Oncol Biol Phys. 2009; 73:935-943. [PubMed: 19111401]

57. Yan, D. On-line adaptive strategy for dose per fraction design.. Presented at the the XIIIth International Conference on the Use of Computers in Radiotherapy; Heidelberg, Germany. 2000; p. 518-520.

58. Lagendijk J, Raaijmakers AJ, Overweg J, et al. MR-XRT at 1.5T, the UMC Utrecht hybrid MRI linac system (abstr). Med Phys. 2009; 36:2775.

59. Chao KK, Goldstein NS, Carlos E. Clinico-pathologic analysis of extra-capsular extension in prostate cancer: Should the clinical target volume be expanded posterior-laterally to account for microscopic extension? Int J Radiat Oncol Biol Phys. 2006; 65:999-1007. [PubMed: 16750320] 

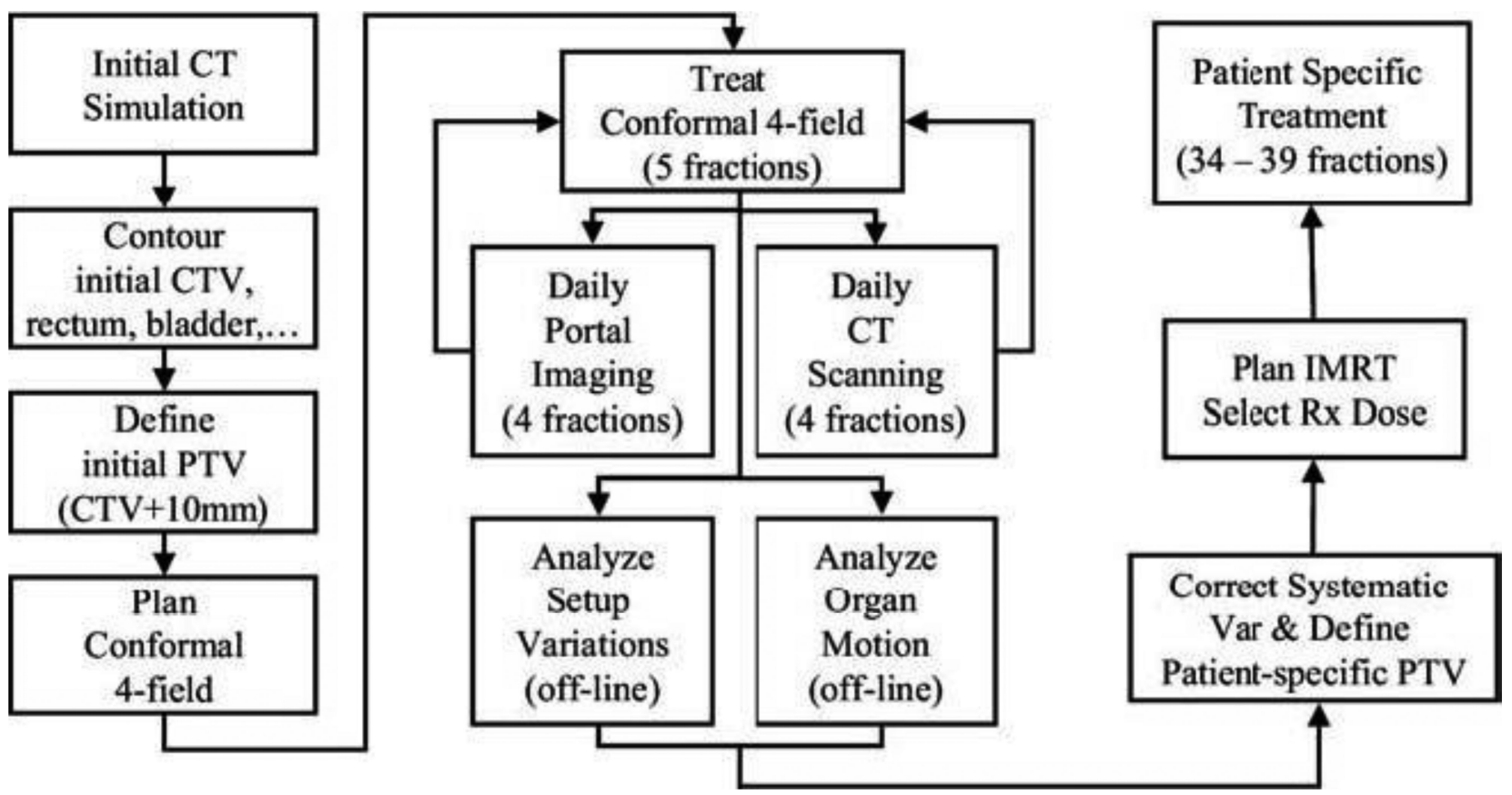

Figure 1.

Flow chart depicting the off-line image guided adaptive radiation therapy process. 


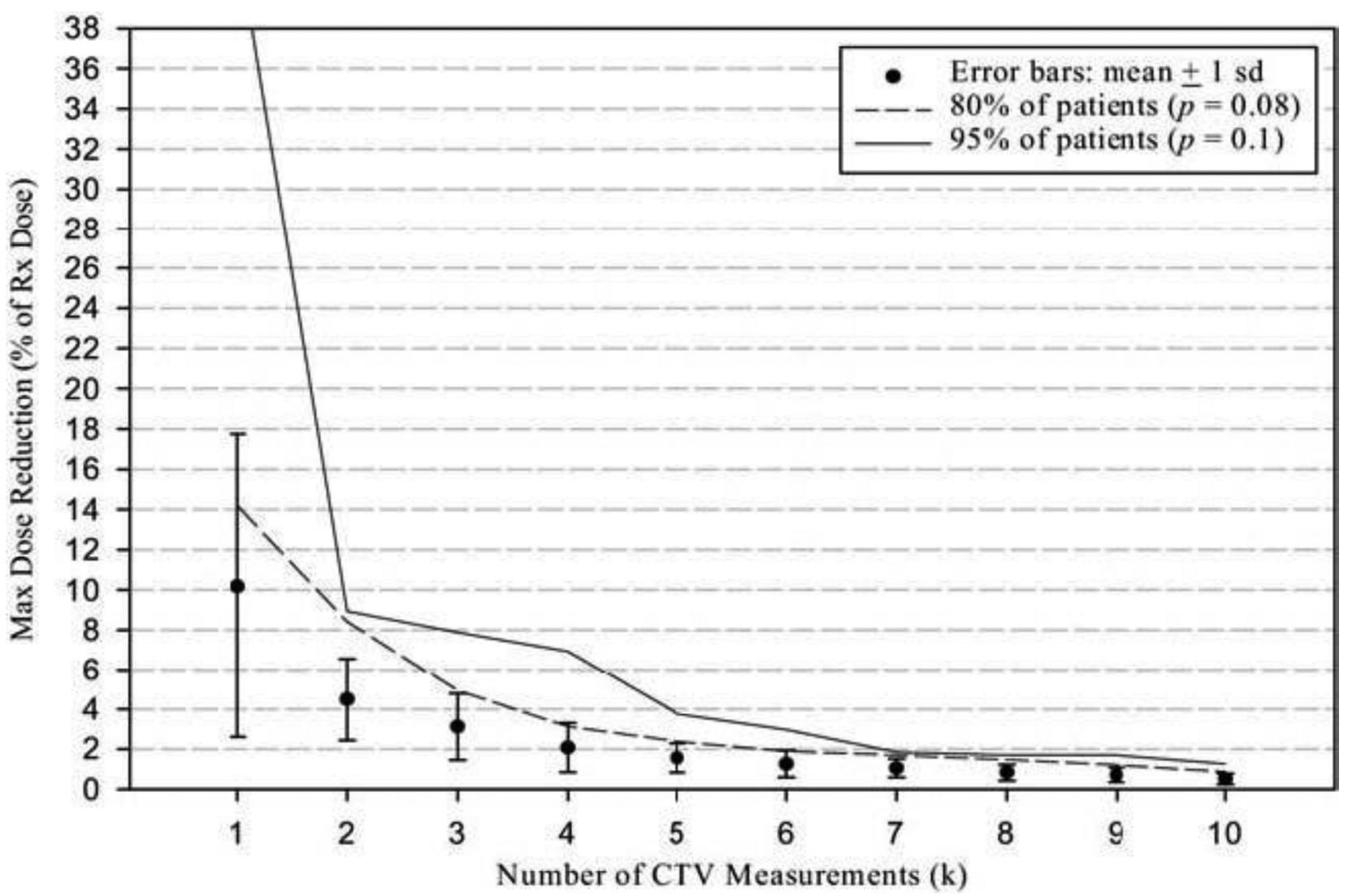

Figure 2.

Error bar indicates the mean and 1 standard deviation of maximum dose reduction for a given number of computed tomography measurements. The maximum dose reduction (\% of Rx. dose) in the clinical target volume due to internal target motion when the patient specific target was applied was estimated considering the worst situation. 


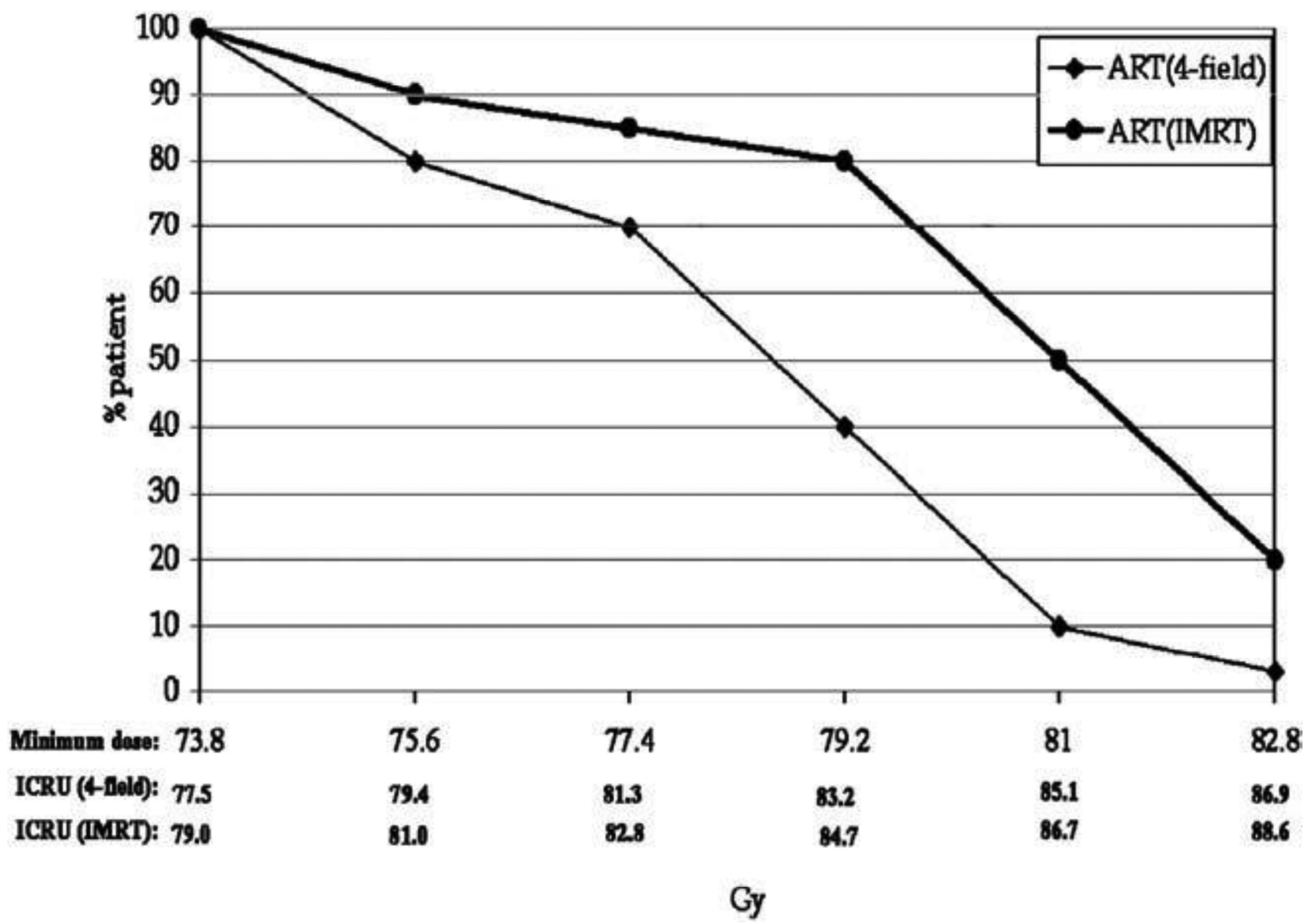

Figure 3.

Distribution of the prescription dose for the patients treated using either a 4-field conformal radiation therapy or a 5-beam intensity-modulated radiation therapy in the off-line adaptive process. 


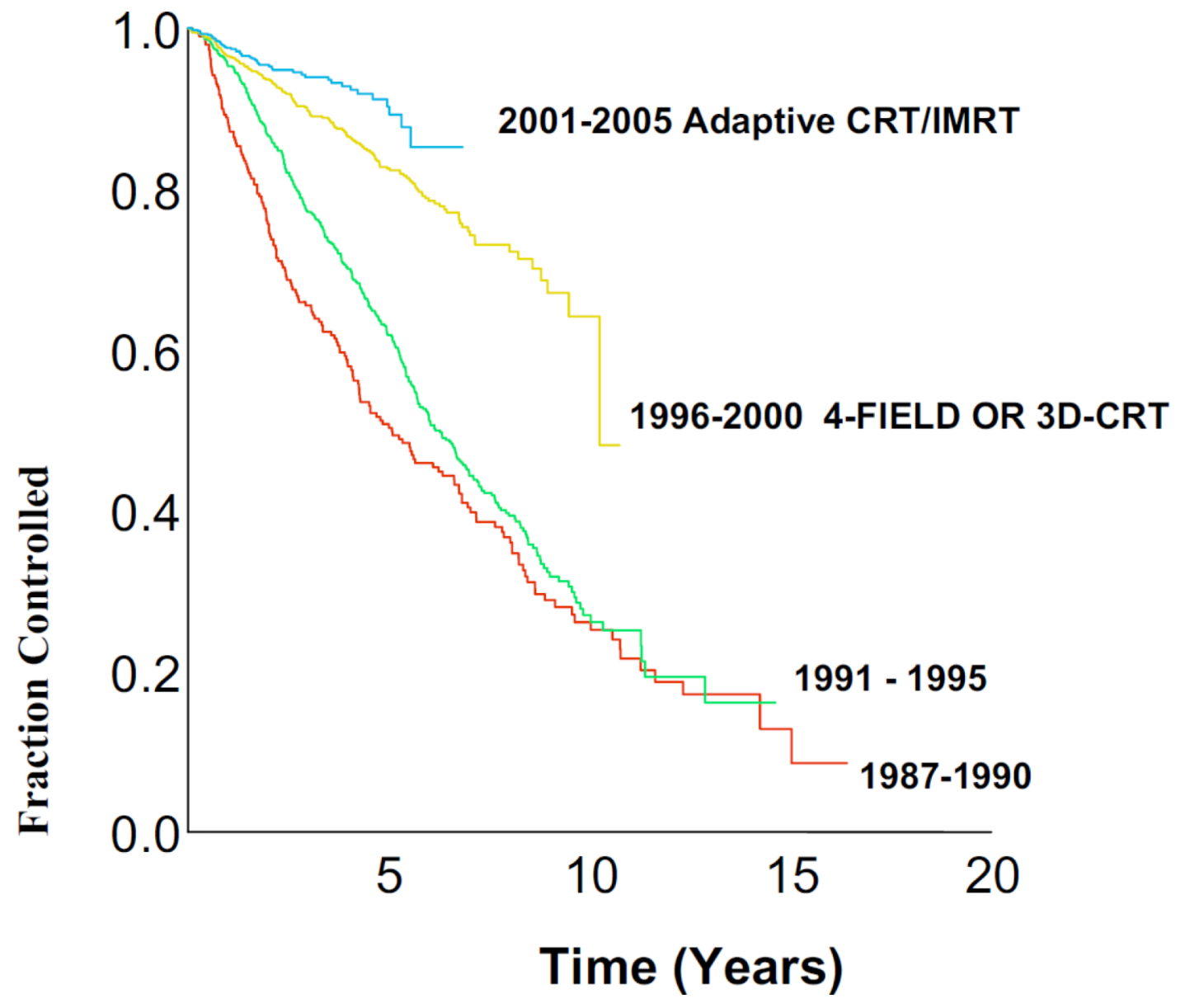

Figure 4.

Actuarial Phoenix Biochemical Control (nadir $+2 \mathrm{ng} / \mathrm{mL}$ ). 
Table 1

Grade 2 Chronic Toxicity by Dose Level

\begin{tabular}{|l|c|c|c|}
\hline & $>\mathbf{7 0 . 2}-<\mathbf{7 2 . 0}$ & $>\mathbf{7 2 . 0}-<\mathbf{7 5 . 6}$ & $>\mathbf{7 5 . 6}-<\mathbf{7 9 . 2}$ \\
\hline Dose Level (Gy) & $(\mathbf{n}=\mathbf{1 0 9})$ & $(\mathbf{n}=\mathbf{3 0 1})$ & $(\mathbf{n}=\mathbf{2 3 2})$ \\
\hline Urinary incontinence & $0 \%$ & $0 \%$ & $0 \%$ \\
\hline Urinary retention & $4 \%$ & $1 \%$ & $2 \%$ \\
\hline Increased frequency/urgency & $0 \%$ & $0 \%$ & $0 \%$ \\
\hline Urethral stricture & $2 \%$ & $0 \%$ & $1 \%$ \\
\hline Hematuria & $3 \%$ & $0 \%$ & $0 \%$ \\
\hline Diarrhea & $0 \%$ & $0 \%$ & $0 \%$ \\
\hline Rectal pain/tenesmus & $0 \%$ & $0 \%$ & $0 \%$ \\
\hline Rectal bleeding & $8 \%$ & $1 \%$ & $2 \%$ \\
\hline Rectal ulceration & $0 \%$ & $0 \%$ & $0 \%$ \\
\hline Rectal fistula & $0 \%$ & $0 \%$ & $0 \%$ \\
\hline Proctitis & $2 \%$ & $0 \%$ & $1 \%$ \\
\hline Rectal incontinence & $0 \%$ & $0 \%$ & $0 \%$ \\
\hline
\end{tabular}


Table 2

Grade 3 Chronic Toxicity by Dose Level

\begin{tabular}{|l|c|c|c|}
\hline & $>\mathbf{7 0 . 2}-<\mathbf{7 2 . 0}$ & $>\mathbf{7 2 . 0}-<\mathbf{7 5 . 6}$ & $>\mathbf{7 5 . 6}-<\mathbf{7 9 . 2}$ \\
\hline Dose Level (Gy) & $(\mathbf{n}=\mathbf{1 0 9})$ & $(\mathbf{n}=\mathbf{3 0 1})$ & $(\mathbf{n}=\mathbf{2 3 2})$ \\
\hline Urinary incontinence & $0 \%$ & $0 \%$ & $0 \%$ \\
\hline Urinary retention & $4 \%$ & $1 \%$ & $2 \%$ \\
\hline Increased freq/urgency & $0 \%$ & $0 \%$ & $0 \%$ \\
\hline Urethral stricture & $2 \%$ & $0 \%$ & $1 \%$ \\
\hline Hematuria & $3 \%$ & $0 \%$ & $0 \%$ \\
\hline Diarrhea & $0 \%$ & $0 \%$ & $0 \%$ \\
\hline Rectal pain/tenesmus & $0 \%$ & $0 \%$ & $0 \%$ \\
\hline Rectal bleeding & $8 \%$ & $1 \%$ & $2 \%$ \\
\hline Rectal ulceration & $0 \%$ & $0 \%$ & $0 \%$ \\
\hline Rectal fistula & $0 \%$ & $0 \%$ & $0 \%$ \\
\hline Proctitis & $2 \%$ & $0 \%$ & $1 \%$ \\
\hline Rectal incontinence & $0 \%$ & $0 \%$ & $0 \%$ \\
\hline
\end{tabular}


Table 3

Toxicity in 728 Patients Treated With Adaptive CRT/ IMRT

\begin{tabular}{|r|c|c|c|}
\hline & Adaptive CRT & Adaptive IMRT & $\boldsymbol{p}$ \\
\hline Acute & Grade 2 +3 & Grade 2 +3 & \\
\hline GU frequency/urgency & $34 \%$ & $30 \%$ & 0.29 \\
\hline Dysuria & $5 \%$ & $2 \%$ & 0.15 \\
\hline GU incontinence & $0.5 \%$ & $2 \%$ & 0.04 \\
\hline Urinary retention & $7 \%$ & $2 \%$ & 0.03 \\
\hline Rectal pain/tenesmus & $19 \%$ & $5 \%$ & $<0.01$ \\
\hline Diarrhea & $10 \%$ & $8 \%$ & 0.43 \\
\hline Chronic & & & \\
\hline GU frequency/urgency & $12 \%$ & $8 \%$ & 0.12 \\
\hline Urinary retention & $3 \%$ & $0.5 \%$ & 0.05 \\
\hline Hematuria & $4 \%$ & $5 \%$ & 0.43 \\
\hline Urethral stricture & $1 \%$ & $2 \%$ & 0.10 \\
\hline Rectal pain/tenesmus & $1 \%$ & $0 \%$ & 0.16 \\
\hline Diarrhea & $3 \%$ & $2 \%$ & 0.51 \\
\hline Rectal bleeding & $16 \%$ & $4 \%$ & $<0.01$ \\
\hline & & & \\
\hline
\end{tabular}


Table 4

5-Year Actuarial Rates

\begin{tabular}{|c|c|c|c|c|}
\hline & \multirow{2}{*}{ All Patients (n = 642) } & Group I & Group II & \multirow{2}{*}{$\boldsymbol{p}$} \\
\cline { 3 - 4 } & & $(\mathbf{n}=\mathbf{3 4 2})$ & $(\mathbf{n = 3 0 0})$ & \\
\hline OS & $87.4 \%$ & $90.2 \%$ & $83.1 \%$ & 0.019 \\
\hline CSS & $97.9 \%$ & $98.7 \%$ & $96.8 \%$ & 0.170 \\
\hline DFS & $90.2 \%$ & $93.9 \%$ & $85.1 \%$ & 0.001 \\
\hline BF & $8.4 \%$ & $5.1 \%$ & $12.8 \%$ & 0.001 \\
\hline
\end{tabular}

